\title{
A study on improving close and distant device movement pose manipulation for hand-held augmented reality
}

\author{
Ali Samini and Karljohan Lundin Palmerius
}

Conference Publication

\section{Tweet}

N.B.: When citing this work, cite the original article.

Original Publication:

Ali Samini and Karljohan Lundin Palmerius, A study on improving close and distant device movement pose manipulation for hand-held augmented reality, VRST '16 Proceedings of the 22nd ACM Conference on Virtual Reality Software and Technology, 2016. (), pp.121-128. http://dx.doi.org/10.1145/2993369.2993380

Copyright: www.acm.org

Postprint available at: Linköping University Electronic Press

http://urn.kb.se/resolve?urn=urn:nbn:se:liu:diva-132954

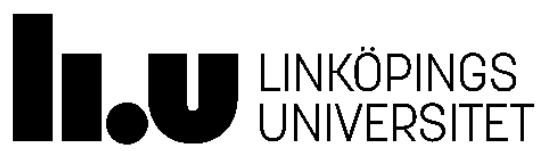




\title{
A Study on Improving Close and Distant Device Movement Pose Manipulation for Hand-Held Augmented Reality
}

\author{
Ali Samini* Karljohan Lundin Palmerius ${ }^{\dagger}$ \\ Department of Science and Technology, \\ Linköping University, Sweden
}

\begin{abstract}
Hand-held smart devices are equipped with powerful processing units, high resolution screens and cameras, that in combination makes them suitable for video see-through Augmented Reality. Many Augmented Reality applications require interaction, such as selection and 3D pose manipulation. One way to perform intuitive, high precision 3D pose manipulation is by direct or indirect mapping of device movement.
\end{abstract}

There are two approaches to device movement interaction; one fixes the virtual object to the device, which therefore becomes the pivot point for the object, thus makes it difficult to rotate without translate. The second approach avoids latter issue by considering rotation and translation separately, relative to the object's center point. The result of this is that the object instead moves out of view for yaw and pitch rotations.

In this paper we study these two techniques and compare them with a modification where user perspective rendering is used to solve the rotation issues. The study showed that the modification improves speed as well as both perceived control and intuitiveness among the subjects.

Concepts: •Human-centered computing $\rightarrow$ Mixed / augmented reality; User studies; Empirical studies in HCI; Gestural input; Usability testing; •Computing methodologies $\rightarrow$ Mixed / augmented reality;

Keywords: device interaction, augmented reality, video seethrough, , user-perspective, device perspective, user study

\section{Introduction}

Hand-held smart devices like cellphones and tablets are popular today, to a level where they can almost be called omnipresent. Furthermore, most of these devices are equipped with powerful processing units, high resolution screens and cameras that makes them capable for video see-through Augmented Reality (V-AR). Such hand-held V-AR captures the view of the world with the backfacing camera and renders it on the screen of the hand-held device with addition of aligned computer graphics augmentations.

Two perspectives have been developed to display hand-held VAR. The most straightforward, and thus the most commonly used,

\footnotetext{
*e-mail: ali.samini@liu.se

†e-mail:karljohan.lundin.palmerius@liu.se
}

Permission to make digital or hard copies of all or part of this work for personal or classroom use is granted without fee provided that copies are not made or distributed for profit or commercial advantage and that copies bear this notice and the full citation on the first page. Copyrights for components of this work owned by others than ACM must be honored. Abstracting with credit is permitted. To copy otherwise, or republish, to post on servers or to redistribute to lists, requires prior specific permission and/or a fee. Request permissions from permissions@acm.org. (c) 2016 ACM.

VRST '16,, November 02-04, 2016, Garching bei München, Germany

ISBN: 978-1-4503-4491-3/16/11

DOI: http://dx.doi.org/10.1145/2993369.2993380 approach creates a view defined by the view of the back-facing camera, Device Perspective Rendering (DPR) [Squire and Jan 2007][Huynh et al. 2009][Mulloni et al. 2011][Dunser et al. 2012]. The result is a display which has always the same point of view (POV) as the camera, which therefore produces a registration error between the users' view around the screen and what is rendered on the screen. A more recent technique instead creates a view from the users' POV, User Perspective Rendering (UPR) [Hill et al. 2011] [Tomioka et al. 2013]. The perspective is dynamically adjusted to co-register the view on the screen with the world seen around the screen and thereby reduces registration errors.

While some AR applications only visually augments the world other require interaction, such as selection and 3D pose manipulation. There are various techniques for 3D virtual object (VO) manipulation. For hand-held V-AR, where a touch surface is available, a commonly used technique acquires multi-touch gestures to interact with the VOs rendered on the screen [Mossel et al. 2013b][Wagner et al. 2005][Rekimoto 1996][Jung et al. 2012]. However, manipulation by movements of the hand-held device itself [Henrysson et al. 2005][Mossel et al. 2013a] is a strong alternative for pose control, primarily because it can provide direct mapping between control and motion, thereby allowing both intuitive and high precision control in 6 degrees-of-freedom (DoF) pose manipulation. Furthermore, it may reduce fatigue, by allowing continuous two handed action, and avoids occlusion during interaction, since no finger is required in front of the screen, or at least not in front of the manipulated VO.

There are two fundamental approaches to full $6 \mathrm{DoF}$ device movement (DM) interaction. The first approach fixes the VO to the device. This will throughout this paper be called fix-DPR. The VO then moves with the hand-held device as if they are connected via a virtual rod. The center of the rotation for $\mathrm{VO}$ is aligned with the center of the hand-held device. Therefore it is difficult to rotate the VO without moving it, see Fig. 1b. This subsequently makes it increasingly cumbersome to control VOs with an increasing distance to the hand-held device. The second approach applies relative translation and rotation separately to the VO, which throughout this paper will be called relative-DPR. The VO rotates with the same amount as the device but around its own center. This allows the user to rotate a $\mathrm{VO}$ without moving it which subsequently makes it easier to manipulate VOs at a distance. However, with the common device perspective rendering, rotating the hand-held device will make VO to go out of view, see Fig. 1c.

In this paper we suggest the combination of the relative-DPR approach together with the UPR approach, to allow good pose control both close and at a distance, while still avoiding the view problem. The translation and rotation are applied separately to the VO, which subsequently can be rotated without being moved. However, the change in the devices's perspective due to rotation does not effect the UPR view, so the VO will be visible on the screen as long as it is in the user's POV, see figure 1d. Figure 2 illustrates interaction as rotation of a virtual chair with all three techniques using our prototype system.

Previous studies on DM methods have been based on the manipulation of VOs that are located in a reachable position. However, 


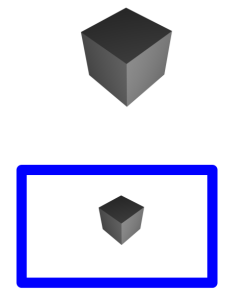

(a)
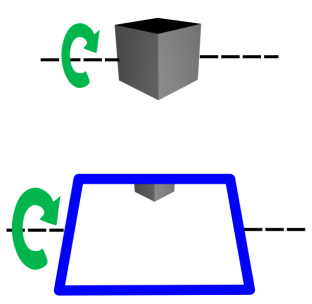

(c)

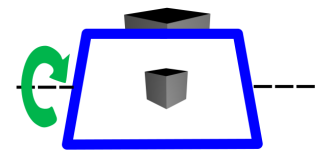

(b)
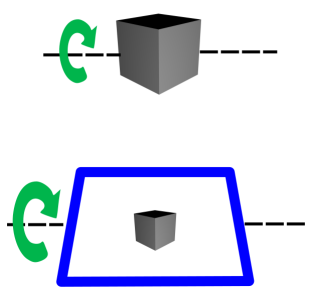

(d)
Figure 1: Device movement interaction, rotation issues. (a) Before manipulation; (b) fix-DPR: VO rotates around the hand-held center. Rotating the device rotates and moves VO; $(c)$ relative-DPR: $V O$ rotates relative to the device. The view rotates with the device therefore VO gets out of the screen; (d) relative-UPR: VO rotates around its own center. The view is created based on the user's perspective therefore $V O$ will stay in the view on the screen after rotating the device.

perceived application of AR include manipulation of both near and far VOs, such as architectural, aerial or even astronomical. Furthermore, during preparations we understood that the fix-DPR technique becomes increasingly unusable as the distance to an unreachable VO increases, due to the long trajectory that the device must be moved to compensate for the translation of the VO during rotation.

We therefore present here our findings from a study on basic DM techniques in DPR and UPR for the manipulation of both near and far VOs. Our goal is to investigate the effect of resolving the rotation issue of the fix-DPR and the visibility issue of the relative-DPR with the application of UPR.

\section{Related Work}

The primary related work for the study presented in this paper are on the areas of user perpective rendering (UPR), and interaction and manipulation using hand-held AR-devices. In fact, we see our proposed relative-UPR DM interaction technique as a combination of a current DM manipulation technique and UPR. Here we first present a background work for the UPR technique, interaction background in AR with the focus on DM-based techniques and then describe our proposed technique in the following section.

\subsection{User Perspective Rendering}

The most common approach to rendering V-AR on hand-held is, because of its straightforward simplicity, the traditional device perspective rendering (DPR) (see for example [Squire and Jan 2007][Huynh et al. 2009][Mulloni et al. 2011][Dunser et al. 2012]). However even though it requires more complicated tracking functionality an increasing amount of work is done with user perspective rendering (UPR). There are two primary techniques for implementing UPR for V-AR: homography-based and projection-based. The former was introduced with ARScope, developed by Yoshida et al.
[Yoshida et al. 2008]. The ARScope application projects the AR view on an opaque surface using a head mounted projector. Their method created a homography from detected feature points in the images captured with a head mounted camera. Hill et al. [Hill et al. 2011] also presented a homography-based method, but theirs used both front and back facing cameras of a hand-held device to implement the required tracking. Their method however assumes a single depth scene parallel to the screen. Tomioka et al. [Tomioka et al. 2013] where successful in creating a homography-based UPR for V-AR using two cameras on a hand-held device.

The projection-based approach was first presented by Samini and Palmerius in [Samini and Palmerius 2014]. They applied Virtual Reality principles to create a geometry projection-based hand-held UPR, using camera-based external tracking.

\subsection{AR Interaction}

Manipulation of VOs in hand-held V-AR is commonly performed using multi-touch interaction. The 2D screen of a hand-held device, however, cannot provide full direct 3D manipulation. Therefore, touch gestures are used to provide manipulation metaphors (see for example [Reisman et al. 2009][Hancock et al. 2009][Martinet et al. 2012][Liu et al. 2012].

Multi-touch interaction is useful, however there are issues affecting the manipulation performance. Performing touch gestures on a 2D screen to do 3D object manipulation can reduce the naturality and intuitiveness of the interaction. Finger occlusion can be another issue: the user's fingers occlude the target on the screen and therefore prevent the target providing a visual feedback. Another issue with the multi-touch is that the user has to hold the device with one hand while press the fingertips of the other hand on the screen. This can be tiresome, specially for larger devices like tablets.

Mid-air gestures can also be used to interact with virtual world. This technique can solve the finger occlusion problem of the touch interaction and have the advantage that user can directly manipulate a VO. Koller et al. [Koller et al. 1996] presented an interaction technique called "head-tracked orbital viewing". The method used head orientation to orbit user's viewpoint on a virtual sphere around a center of rotation. While it is a very interesting method it is unable to move the $\mathrm{VO}$ and also rotating the head for manipulation can be tiresome. Hürst and van Wezel, [Hürst and van Wezel 2013] presented their research on potential of finger tracking for gesturebased interaction through two experiments. Their results indicated a high entertainment value, but low accuracy. The method can be tiresome for bigger screen hand-held devices while the user has to hold the device with one hand and perform the mid air gesture with the other hand.

As an alternative the device itself can be used for 6 DoF tracking and manipulation. This device-based manipulation (DM) was first presented by Henrysson et al. [Henrysson et al. 2005], allowing movements of the hand-held device to be used to manipulate VOs. In this technique changes in the position and orientation of the device is used to change the pose of the VO.

There are two general approaches to DM manipulation in V-AR. The most common approach fixes the VO to the hand-held device (fix-DPR). The VO is translated with the device and is rotated around the device's center. Henrysson et al. [Henrysson et al. 2005] implemented fix-DPR hand-held V-AR manipulation for a Symbian mobile phone. The pose of the device was tracked using a fiducial marker that was visible by its camera. Katzakis and Masahiro [Katzakis and Hori 2009] used a mobile phone as 3 DoF controller to complement mouse and keyboard for changing the orientation of VOs. Du et al. [Du et al. 2011] used combined mobile device 

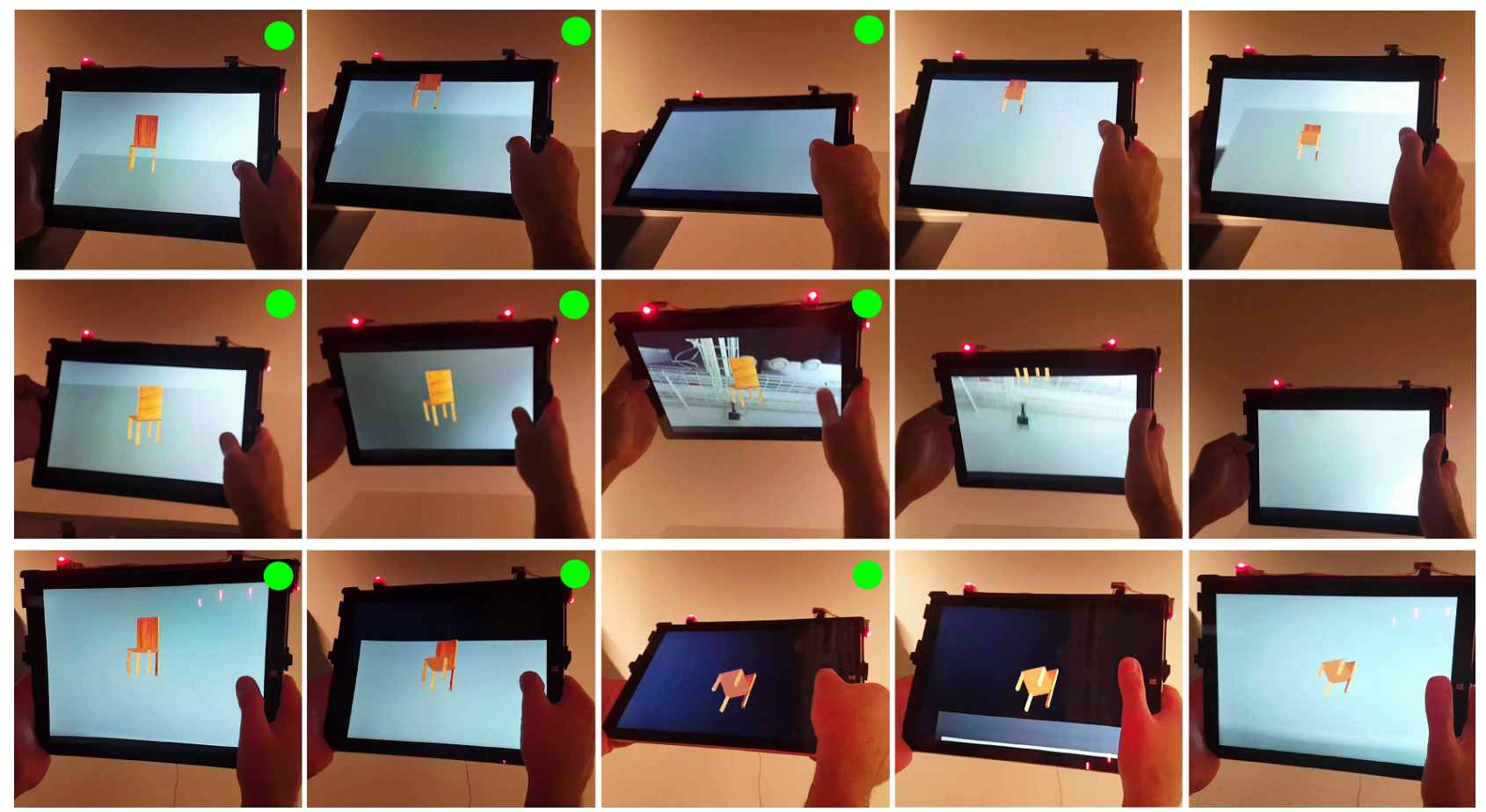

Figure 2: "Rotating a virtual chair using our prototype system. A green circles indicates that the VO is selected thus locked to the device. (first row) Relative-DPR: VO is rotated relative to device. When the tablet is rotated the view is changed and therefore VO is not visible on the screen. (second row) Fix-DPR: VO is locked to the device. Thus rotating the device rotates and also moves the VO. As the result VO is not at the same place as it was before rotation. (third row) Relative-UPR: Vo rotates relative to the device. The view is from user's perspective and thus isn't changed when the device is rotated and VO stays visible on the screen.

movement and touch screen as 3D controller of other large display. The pose of the VO changed based on 3D displacement of the finger on device and the change of orientation of the device. Sasakura et al. [Sasakura et al. 2011] developed a 3D molecular visualization system in which the user can manipulate molecules by mobile devices. They used 3D acceleration sensors of the mobile device to measure device movement and the molecular system was rendered on both the hand-held and a remote screen.

The main issue with fix-DPR is the difficulty of performing rotation without moving the object, see figure $1 \mathrm{~b}$. The second approach instead directly maps the position and orientation of the manipulation device to the VO (relative-DPR). Mossel et al. [Mossel et al. 2013a] developed this in their HOMER-S application, that also allows for separate translation and rotation via mode switches. The issue of relative-DPR is that the VO leaves the view while rotation is performed. This occurs for yaw and pitch rotations and since the direction of the back-facing camera is mostly parallel with the roll axis the perspective is less affected by roll rotations.

Marzo et al. [Marzo et al. 2014] developed a hybrid method to solve the rotation issue of relative-DPR. Device movement is used to translate the VO while multi-touch is used to change the orientation and move the object near and far. This technique takes advantages of both methods, however it retains most of the limitations of the multi-touch technique.

The main problem with the DM techniques stem from the fact the view does not allow the VO to be continuously visible as the device is rotated. We therefore propose a DM method that is the combination of the relative-DPR and the user perspective rendering (relative-UPR). Our technique directly maps the devices pose to the VO in a UPR-based V-AR. We believe this technique solves the main issues with both relative-DPR and fix-DPR. The VO's pivot point is at its center and it will be visible on the screen while rotating as long as it is within the user's POV.

\section{User Study}

In order to evaluate the performance and usability of the proposed hand-held DM interaction technique we conducted a user study. The experiment was designed to compare two existing DM techniques, fix-DPR and relative-DPR, with the UPR technique proposed in this paper. Because of the distance related issues with the DPR methods, the study was divided into two separate tasks: one with a near VO with which the subjects could move around it and both view and interact from any angle, and the other with a VO that was placed at an unreachable distance from the subject. While the former was performed with all three techniques, the latter could only be performed with relative-DPR and relative-UPR. This was since the fix-DPR, as mentioned in the introduction, becomes increasingly difficult to use with an increasing distance to VO. Dividing the study also gave us the opportunity to investigate if DM techniques perform differently relative to the VO's distance.

\subsection{Relative Control with UPR}

Our proposed technique is a combination of the relative manipulation applied in relative-DPR and user perspective rendering of the AR view. This should remove the most prominent issues with this approach, which we believe is the fact that the VO moves out of view as the user applies rotation.

In our implementation manipulation of the VO is started with selection that is done by touching any place on the screen (Fig. 3b). 


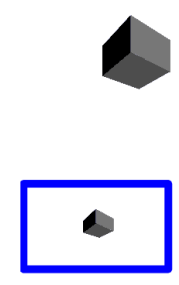

(a)

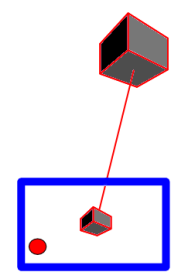

(b)

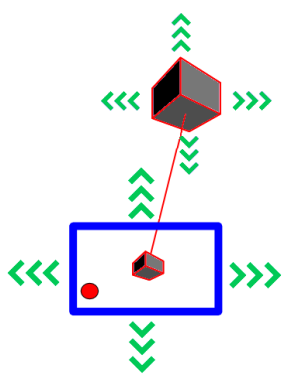

(c)

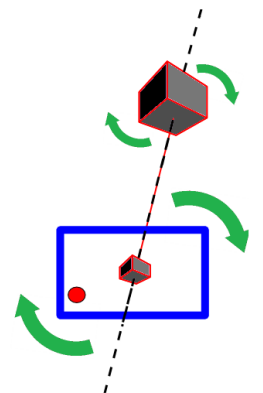

(d)

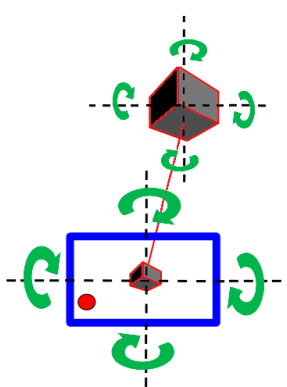

(e)

Figure 3: relative-UPR DM interaction: (a) The VO is visible on the screen; (b) the VO is selected by touching anywhere on the screen; (c) moving the device translates the VO relatively in the same direction; $(d)$ roll; $(e)$ yaw and pitch rotations of the device creates same rotations for VO around its local axis.

Another application can instead have the user touch the object itself to initiate manipulation. Translating the hand-held device will translate VO relatively in the virtual world (Fig. 3c), and rotating the device will rotate the VO around VO's center relatively (Fig. 3d and $3 \mathrm{e}$ ). The VO will stay selected while the user continues to touch the screen.

The orientation and translation of the manipulated VO at each frame is calculated based on the poses of the device and the VO when the VO was first locked to the device and also the current pose of the device. The current orientation of the $\mathrm{VO}, r_{\mathrm{o}}$, is calculated as

$$
r_{o 2}=r_{s 2} r_{s o}
$$

where $r_{s 2}$ is the current orientation of the screen and $r_{s o}$ is the fixed relative orientation of $\mathrm{VO}$ and the screen, which is calculate at locking time as,

$$
r_{s o}=\operatorname{inv}\left(r_{s 1}\right) r_{o 1}
$$

where $r_{s 1}$ and $r_{o 1}$ are the orientations of the screen and the $\mathrm{VO}$ at locking instant, respectively. Likewise, the new VO position, $t_{o 2}$, is calculated as,

$$
t_{o 2}=t_{o 1}+\left(t_{s 2}-t_{s 1}\right)
$$

where $t_{o 1}$ and $t_{s 1}$ are the positions of the screen and VO at locking instant and $t_{s 2}$ is the current position of the screen.

Using UPR for the manipulation technique applied in fix-DPR is also fully possible, however it does not solve any issues as far as we can see, while introducing new issues, and is therefore neither proposed as a viable solution nor tested in this study.

\subsection{Expected Outcome and Hypotheses}

The expected outcome from the study was based on a preliminary analysis of advantages and drawbacks of each technique. We believe that the main problem with relative-DPR is for yaw and pitch rotations, which cause the VO to get out of view. The fix-DPR method solves that problem and keeps the selected VO always visible, however subsequently performing rotations also moves the VO.

Relative-UPR solves the rotation problem of relative-DPR. The view does not change with the orientation of the hand-held device and therefore the VO will be visible on the screen as long as it is within the user's view. Furthermore, relative-UPR separates the rotation and translation of $\mathrm{VO}$ so it is possible to rotate the VO without moving it, as opposed to with fix-DPR.
Based on these observations, we formulated our hypotheses as follows:

H1: Subjects will perform the 3D docking task for a near $V O$ faster with relative-UPR compared to relative-DPR.

H2: Subjects will perform the 3D docking task for a near $V O$ faster with relative-UPR compared to fix-DPR.

H3: Subjects will perform the 3D docking task for a far $V O$ faster with relative-UPR compared to relative-DPR.

We also collected meta information about independent states to allow for further analysis on the study data, complementing the core findings. Such partial analysis typically leave too few repetitions for reliable statistical analysis and can also increase the problem of multiple comparisons. Therefore, statistical significance is not considered for the further analysis and is also not included in the Bonferroni compensations.

\subsection{Experimental Design}

The study was designed to compare DM-based interaction techniques using a 6 DoF docking task. The task was to move and align 3D model of a chair (cursor) with a wireframe model of the same chair (target). The independent variables were the interaction techniques and the dependent variable was performance in the form of the time it took to complete the task.

We divided the test into two separate tasks based on the distance between the test subjects and the cursor/target. In the first task the cursor and the target both were placed near the subject within a reachable area. In the second task the cursor and target were placed so far from the subject that made it impossible to go around them and view from various angles. The subjects performed the first test using fix-DPR, relative-DPR, and relative-UPR. However, as mentioned before, the far test was only performed with relative-DPR and relative-UPR.

The near test was done with a $10 \times 4 \times 4$ centimeters virtual chair. The target was placed on a $1.5 \times 1.1$ meters table at the center, 1.2 meters above the floor. Each subject did twenty dockings per technique, $20 \times 3=60$ docking tasks in total. Each docking started with a cursor pose that was randomized between a pre-defined set.

The position and orientation offset of the cursor relative to the target for the near test were carefully designed and saved as meta data for further analysis of the results. The cursor poses at the start of a task was categorized into four modes: translation-only, rotationonly, small-rotation and large-rotation. Positions in the translation- 


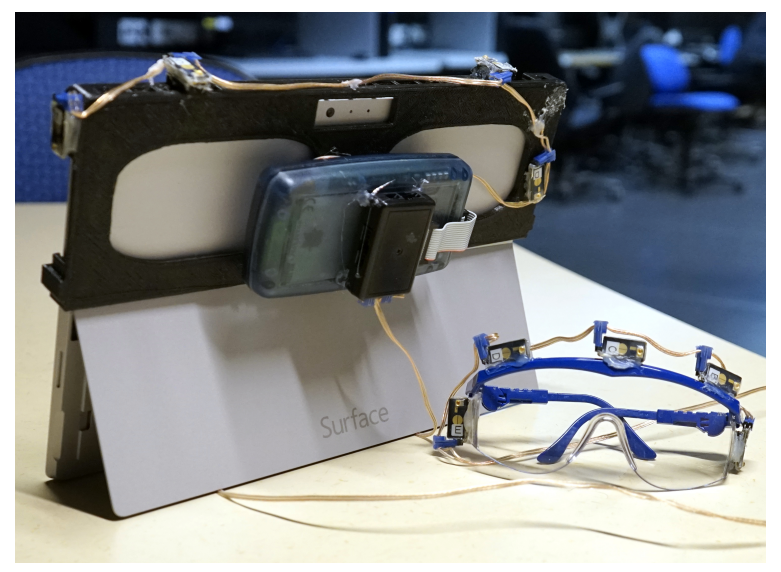

Figure 4: The apparatus: A tablet PC covered with a $3 D$ printed frame that is the housing for the illuminative markers and the LED power-source and unit driver. A pair of safety goggles attached with illuminative markers.

only group were randomized on a balanced set between 10 and 30 centimeters from the target while the orientations were same as the target. The rotation-only group had orientations that was randomized on a balanced set between 120 and 150 degrees around each axis relative to the target. The positions of this group were same as the target. The small-rot and large-rot groups included orientations randomized on a balanced set in the range of 30 to 60 degrees and 120 to 150 degrees relatively, around each axis relative to the target. Both latter groups had translations randomized between 10 to 30 centimeters from the target.

In the far test the docking was performed with the target placed 10 meters away from the subject, on the floor. The size of the virtual model was chosen to be the same as a real chair. For each technique 10 dockings were required to complete the test, $10 \times 2=20$ docking in total. The relative pose of the cursor and the target was randomized on balanced sets between 2 to 4 meters for translation and 120 and 150 degrees around each axis for rotation.

The subjects could select and grab the VO by touching anywhere on the screen, to avoid selection having an impact on the final results. Also recorded as meta information was the number of times the subject released the VO to change pose. This is necessary when a large rotation is required to match the cursor with the target, however can also be made by the subject to move the device into a more comfortable orientation.

\subsection{Test Environment and Prototype}

The test was performed in an area of $2 \times 2$ meters covered by a PhaseSpace LED-based optical motion tracking system for 6 DoF realtime tracking. An off-the-shelf Microsoft Surface Pro 3 tablet PC was used as hand-held V-AR device and also for standalone, online processing, and the back-side camera was used to capture live V-AR video feed. A 3D printed frame was attached to the tablet, serving as housing for the LEDs, LED power-source and LED driver. A pair of safety goggles also equipped with LED markers was used for head tracking, see Fig. 4.

VRPN [Taylor et al. 2001] was used to communicate tracking data to the tablet PC. C++, OpenGL and SDL [SDL ] were used for the software. The camera was calibrated and the live view was captured and undistorted using OpenCV.

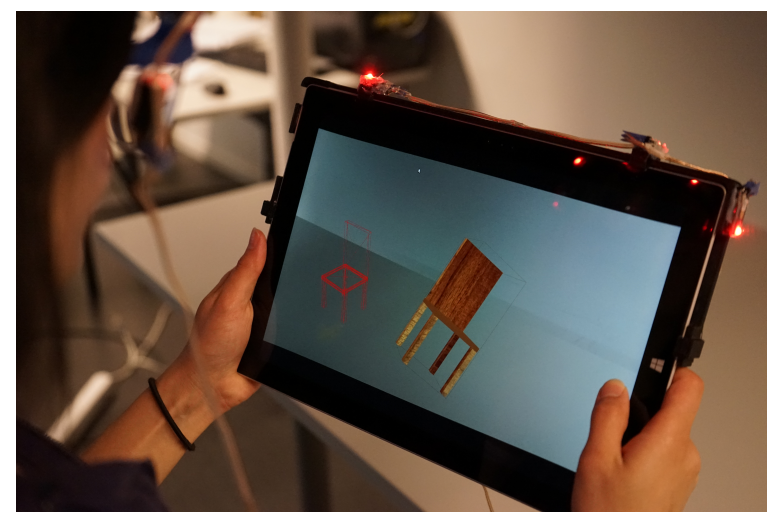

Figure 5: A test participant performing the 3D docking task for a near $V O$.

\subsection{Participants and Procedure}

The study was performed with 15 subjects ( 5 female and 10 male) recruited from university with age ranging between 26 and 40 $(M d n=30)$. To minimize the impact of learning effects, the order of used techniques in the test was balanced as far as possible with the 15 subjects that took the near test. For the far test 14 subjects from the same group were used to balance the order completely. We found no physical disability nor uncorrected vision problem that were expected to affect the test. All subjects reported to be experienced with hand-held smart devices, 10 subjects were familiar with $\mathrm{AR}$ and 5 reported to be familiar with UPR.

At the start subjects were given a pre-test questionnaire to input their general information, physical disabilities and level of experience with the used techniques. Upon completion the DM interaction and the used techniques were thoroughly explained to subjects by a tutor. Then subjects were trained and practiced each task and only started the actual test when they felt confident with the techniques. The subjects wore the tracked goggles, held the tablet device and started each task by touching the screen. The test data was recorded from when the subjects touched the start screen. During each task a new VO appeared automatically after the last one was docked. The subject was notified with an on screen message when the task was finished.

For the near test subjects started standing in front of a table, above which both the cursor and target appeared. The subjects could go around the table during the test to see the VO from different angles. For the far test the cursor and target were presented at a distance and the subjects could not go around them. Figure 5 shows a subject performing the near 3D docking task.

Each subject did 5 separate docking tasks, 3 near with 20 dockings each and 2 far with 10 dockings each, using the DM techniques. After performing all tasks the subjects were given a questionnaire to capture their overall preference of the used techniques. The test took approximately 50 minutes for each participant.

\section{Results}

For the near test, we used the Kolmogorov-Smirnov test to verify that the performance data were normally distributed. Single-factor repeated measures ANOVA, at $\mathrm{p}<0.05$, was conducted to compare the effect of using different DM techniques on the mean task completion time. Post hoc test with Bonferroni correction was used in case of statistical significance. Greenhouse-Geisser correction was applied whenever the sphericity condition was violated. 
Table 1: Descriptive statistics of the near test. The mean is the task completion time for all $(N)$ users in seconds.

\begin{tabular}{llll} 
Technique & Mean & Std. Deviation & $\mathrm{N}$ \\
\hline Relative-DPR & 17.884 & 7.014 & 15 \\
Fix-DPR & 13.86 & 4.245 & 15 \\
Relative-UPR & 11.745 & 4.576 & 15
\end{tabular}

Table 2: Descriptive statistics of the far test. The mean is the task completion time for all $(N)$ users in seconds.

\begin{tabular}{llll} 
Technique & Mean & Std. Deviation & N \\
\hline Relative-DPR & 16.719 & 6.457 & 14 \\
Relative-UPR & 10.467 & 3.138 & 14
\end{tabular}

The data from the far test were analysed with one tail t-test to compare the task completion time. The direction of the one-tail test was defined based on our previously made hypothesis.

\subsection{Near Test}

Table 1 shows descriptive statistics of the near test. The task completion time differed statistically significantly between the used techniques $(\mathrm{F}(2,13)=7.739, \mathrm{p}=0.006)$. Post hoc tests revealed that the mean task completion time for relative-UPR was significantly faster than relative-DPR ( $\mathrm{p}=0.004)$. There was no significant difference between the mean task completion time of relative-UPR and fix-DPR $(\mathrm{p}=0.092)$.

The results support $\mathrm{H} 1$ that stated the relative-UPR will be faster than relative-DPR, so H1 is accepted. Our second hypothesis, H2, is not confirmed, since there where no statistically significant difference between the task completion time for fix-DPR and relativeUPR.

\subsection{Far Test}

The descriptive statistics of the far test are shown in Table 2. One tail t-test $\left(\mathrm{t}(13)=4.068, \mathrm{p}=7 \times 10^{-4}\right)$ shows statistical significance between the task completion time for relative-DPR $(M=16.719$, $\mathrm{SD}=6.275)$ and relative-UPR $(\mathrm{M}=10.467, \mathrm{SD}=4.0146)$, in the favor of relative-UPR.

The result supports $\mathrm{H} 3$ that stated that relative-UPR is significantly faster than relative-DPR to interact with a far VO.

\subsection{Further Analysis}

The mean task completion time of the subjects in the near interaction test using each method are shown in Fig. 6. The same data for the far test are shown in Fig. 7.

For further analysis of the data, the same statistical methods were applied as for the hypotheses falsification process descried earlier, but here for each mode separately (translation-only, rotation-only, small-rotation and large-rotation). Analysing the task completion time for the translation-only mode showed that the difference seen is not unprobable to have arisen by chance $(\mathrm{p}=0.127)$. That is understandable considering that translation manipulation for the compared techniques is mostly similar. The difference in mean task completion time for the rotation-only mode is less probable to have arisen by chance: the data indicate that relative-UPR is faster than relative-DPR $(\mathrm{p}=0.003)$ and that relative-UPR is faster than fixDPR $(p=0.037)$. The mean task completion time differences for the small-rotation and large-rotation modes is less probable to have

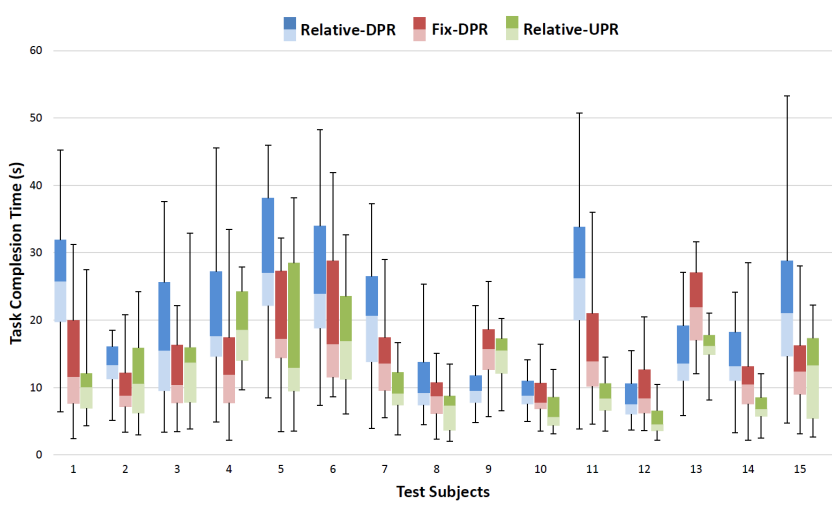

Figure 6: The task completion time of the near test for relative$D P R, f i x-D P R$, and relative-UPR techniques showing min, median and max for each participant using each technique. Quartiles 2 and 3 are indicated with lighter and darker colors, respectively.

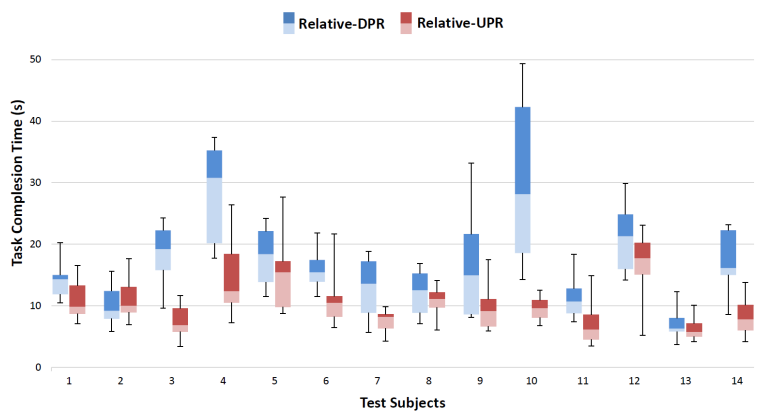

Figure 7: The task completion time of the far test for relative-DPR and relative-UPR techniques showing min, median and max for each participant using each technique. Quartiles 2 and 3 are indicated with lighter and darker colors, respectively.

arisen by chance between relative-DPR and relative UPR. The data indicates that relative-UPR is faster than relative-DPR for smallrotation $(p=0.004)$ and large-rotation $(p=0.024)$ modes. However the differences between fix-DPR and relative-UPR are not unprobable to be arisen from chance for small-rotation $(\mathrm{p}=0.526)$ and largerotation $(\mathrm{p}=0.234)$ modes.

The number of times the subject released the VO to change pose was also compared for both the near and far tests, shown in Table 3. The data show a strong indication that relative-UPR may require less pose changes than both relative-DPR $\left(p=3 \times 10^{-7}\right)$ and fixDPR $(\mathrm{p}=0.012)$ in near interaction as well as in far interaction with relative-DPR $\left(\mathrm{p}=6 \times 10^{-6}\right)$.

Table 3: Descriptive statistics of the number of times the subject released the VO to change pose during the docking task. The mean is of the number of pose changes over all $(N)$ users.

\begin{tabular}{|c|c|c|c|}
\hline Technique & Mean & Std. Deviation & $\mathrm{N}$ \\
\hline Relative-DPR & 113.6 & 28.493 & 15 \\
\hline Fix-DPR & 55.73 & 6.974 & 15 \\
\hline Relative-UPR & 41.467 & 14.141 & 15 \\
\hline \multirow{2}{*}{$\begin{array}{ll}\text { Relative-DPR } \\
\text { 氠 } & \text { Relative-UPR }\end{array}$} & 76.857 & 24.844 & 14 \\
\hline & 33.786 & 12.192 & 14 \\
\hline
\end{tabular}




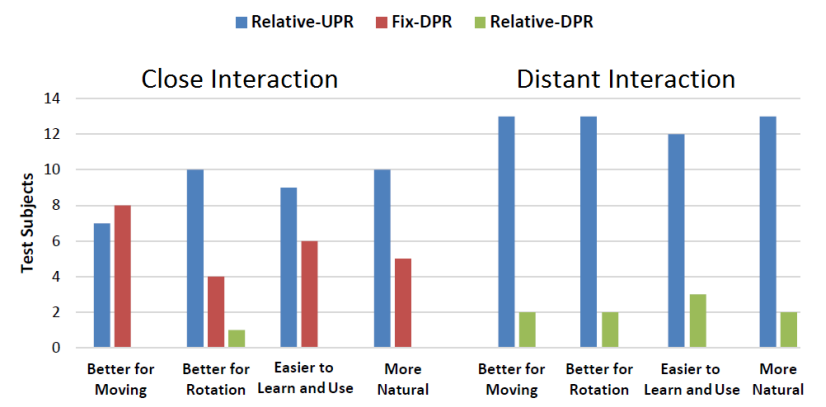

Figure 8: The results from the post-test questionnaire showing subject's over all preference separately for the near and far test.

\subsection{Post-Test Questionnaire}

The post experiment questionnaire was designed to capture the overall preference of the subjects. The questions and answers are listed in Fig. 8. We first asked the subjects which technique they preferred for rotating and which for moving a VO that is placed near or far away. 10 subjects preferred relative-UPR, 4 fix-DPR, and 1 relative-DPR to rotate a near VO. To translate a near VO 7 subjects preferred relative-UPR and 8 fix-DPR and there was no vote for relative-DPR. For a far VO 12 subjects preferred relativeUPR over relative-DPR for both moving and rotation.

The subjects where also asked which technique was easier to learn and to use. 9 subjects found relative-UPR, 6 fix-DPR, and none relative-DPR to be easier than other techniques to perform the near test. For the far test 11 subjects chose relative-UPR and 3 relativeDPR to be the easiest technique.

Finally we asked which technique felt more natural and intuitive during the test. 10 subjects chose relative-UPR and 5 fix-DPR to be more natural for near object manipulation. For a VO 12 chose relative-UPR over relative-DPR to be more natural and intuitive.

\section{Discussion}

The difference between relative-UPR and fix-DPR in rotation-mode reported in section 4.3 can also be observed in the subject specific data of the overall tasks, listed in Fig. 5, however these differences did not yield statistical significance, as reported in section 4.1. We believe that the possible difference between these two techniques for the tasks that only include rotation can be explained by the intrinsic difficulty of rotating without translating with fixDPR method that is solved with relative-UPR.

The data over the number of times the subject released the VO to change pose indicate that relative-UPR requires less such pose changes than the other techniques. Using relative-DPR subjects needed to divide a large rotation to sequential small ones and keep the $\mathrm{VO}$ visible on the screen therefore the technique required more selection. With fix-DPR to avoid moving the tablet on long trajectories performing a large rotation it was also necessary to divide it into small sequential rotations. Relative-UPR keeps a VO that is within the user's perspective on the screen and therefore allows for larger rotations with one single motion.

Also, we believe that more subjects preferred relative-UPR rotation for both near and far tests for the same reason, because of the rotation issues in DPR. The close preference result for translation of a near VO between relative-UPR and fix-DPR can be because of the similar approach the techniques have to move a VO. However, this is somewhat surprising since we believe relative-UPR to be more similar to relative-DPR with respect to how translation is performed.

We also believe more subjects found relative-UPR easier due to the corrected perspective that ease the manipulation. Relative-UPR creates a perspective based on the subject's POV compared to the other techniques that use the device's POV.

\section{Conclusion}

This paper has presented a user study designed to highlight important issues with current techniques for device motion-based pose manipulation in hand-held video see-through Augmented Reality and how these can be reduced by applying User Perspective Rendering (UPR). The study was performed on both near and distant interaction to cover a wide range of applications.

In pose manipulation of reachable objects the subjects prefered relative-UPR, but of the two DPR-based techniques fix-DPR was preferred. This, however, was the slowest technique with statistical significance $(\mathrm{p}=0.006)$. Relative-UPR was not found to be faster than relative-DPR $(\mathrm{p}=0.092)$, however the data indicate that while they may be similar in performance for the translation component of the tasks, relative-UPR seems to be faster for the rotation component. This also matches the initial analysis of the interaction techniques.

In pose manipulation of distant objects the subjects prefered relative-UPR over relative-DPR. Analysis and pilot with fix-DPR showed that it is unusable in its pure form for manipulation at a distance. The numerical analysis also showed that relative-UPR is faster, with statistical significance $\left(\mathrm{p}=7 \times 10^{-4}\right)$.

Further anlysis of the data also indicate that the number of times that a user needs to release the virtual object to change pose is less with relative-UPR than with fix-DPR, which in turn is less than with relative-DPR. Observations indicate that this is because the virtual object comes increasingly fast out-of-view in that order, when performing rotation.

\section{Acknowledgements}

This work has been supported by SSF through a grant for the project CUAS. The authors would also like to thank Camilla Forsell for valuable input on this paper, and the staff at the Indoor Micro Unmanned Aerial Vehicle Laboratory.

\section{References}

Du, Y., Ren, H., Pan, G., AND Li, S. 2011. Tilt \& touch: Mobile phone for 3d interaction. In Proceedings of the 13th International Conference on Ubiquitous Computing, ACM, New York, NY, USA, UbiComp '11, 485-486.

Dunser, A., Billinghurst, M., Wen, J., Lehtinen, V., AND NuRminen, A. 2012. Technical section: Exploring the use of handheld ar for outdoor navigation. Comput. Graph. 36, 8 (Dec.), 1084-1095.

Hancock, M., ten Cate, T., and Carpendale, S. 2009. Sticky tools: Full 6dof force-based interaction for multi-touch tables. In Proceedings of the ACM International Conference on Interactive Tabletops and Surfaces, ACM, New York, NY, USA, ITS '09, 133-140.

Henrysson, A., Billinghurst, M., And Ollila, M. 2005. Virtual object manipulation using a mobile phone. In Proceed- 
ings of the 2005 International Conference on Augmented Teleexistence, ACM, New York, NY, USA, ICAT '05, 164-171.

Hill, A., Schiefer, J., Wilson, J., Davidson, B., Gandy, M., AND MACINTYRE, B. 2011. Virtual transparency: Introducing parallax view into video see-through ar. 2013 IEEE International Symposium on Mixed and Augmented Reality (ISMAR) $0,239-240$

HÜRST, W., AND VAN WEZEL, C. 2013. Gesture-based interaction via finger tracking for mobile augmented reality. Multimedia Tools and Applications 62, 1, 233-258.

Huynh, D.-N. T., Raveendran, K., Xu, Y., Spreen, K., And MacIntyre, B. 2009. Art of defense: A collaborative handheld augmented reality board game. In Proceedings of the 2009 ACM SIGGRAPH Symposium on Video Games, ACM, New York, NY, USA, Sandbox '09, 135-142.

Jung, J., Hong, J., PARK, S., And YAng, H. S. 2012. Smartphone as an augmented reality authoring tool via multi-touch based 3d interaction method. In Proceedings of the 11th ACM SIGGRAPH International Conference on Virtual-Reality Continuum and Its Applications in Industry, ACM, New York, NY, USA, VRCAI '12, 17-20.

KATZAKIS, N., AND Hori, M. 2009. Mobile phones as 3-dof controllers: A comparative study. In Dependable, Autonomic and Secure Computing, 2009. DASC '09. Eighth IEEE International Conference on, 345-349.

Koller, D. R., Mine, M. R., And Hudson, S. E. 1996. Headtracked orbital viewing: An interaction technique for immersive virtual environments. In Proceedings of the 9th Annual ACM Symposium on User Interface Software and Technology, ACM, New York, NY, USA, UIST '96, 81-82.

LiU, J., AU, O. K.-C., Fu, H., AND TAI, C.-L. 2012. Two-finger gestures for $6 \mathrm{dof}$ manipulation of $3 \mathrm{~d}$ objects. Comput. Graph. Forum 31, 7pt1 (Sept.), 2047-2055.

Martinet, A., CAsiez, G., AND Grisoni, L. 2012. Integrality and separability of multitouch interaction techniques in $3 \mathrm{~d}$ manipulation tasks. IEEE Transactions on Visualization and Computer Graphics 18, 3 (Mar.), 369-380.

Marzo, A., Bossavit, B., And Hachet, M. 2014. Combining multi-touch input and device movement for $3 \mathrm{~d}$ manipulations in mobile augmented reality environments. In Proceedings of the $2 N d$ ACM Symposium on Spatial User Interaction, ACM, New York, NY, USA, SUI '14, 13-16.

Mossel, A., Venditti, B., And Kaufmann, H. 2013. 3dtouch and homer-s: Intuitive manipulation techniques for one-handed handheld augmented reality. In Proceedings of the Virtual Reality International Conference: Laval Virtual, ACM, New York, NY, USA, VRIC '13, 12:1-12:10.

Mossel, A., Venditti, B., And Kaufmann, H. 2013. Drillsample: precise selection in dense handheld augmented reality environments. In Proceedings of the Virtual Reality International Conference: Laval Virtual, ACM, 10.

Mulloni, A., Seichter, H., and Schmalstieg, D. 2011. Handheld augmented reality indoor navigation with activitybased instructions. In Proceedings of the 13th International Conference on Human Computer Interaction with Mobile Devices and Services, ACM, New York, NY, USA, MobileHCI '11, 211220.

Reisman, J. L., Davidson, P. L., And Han, J. Y. 2009. A screen-space formulation for $2 \mathrm{~d}$ and $3 \mathrm{~d}$ direct manipulation. In
Proceedings of the 22Nd Annual ACM Symposium on User Interface Software and Technology, ACM, New York, NY, USA, UIST '09, 69-78.

Rekimoto, J., 1996. Transvision: A hand-held augmented reality system for collaborative design.

SAmini, A., AND PAlmeriUs, K. L. 2014. A perspective geometry approach to user-perspective rendering in hand-held video see-through augmented reality. In Proceedings of the 20th ACM Symposium on Virtual Reality Software and Technology, ACM, New York, NY, USA, VRST'14, 207-208.

Sasakura, M., Kotaki, A., AND InADA, J. 2011. A 3d molecular visualization system with mobile devices. In 2011 15th International Conference on Information Visualisation, 429-433.

SDL: Simple directmedia layer. http://http://www.libsdl.org/.

SQUiRE, K. D., AND JAN, M. 2007. Mad city mystery: Developing scientific argumentation skills with a place-based augmented reality game on handheld computers. Journal of Science Education and Technology 16, 1, 5-29.

Taylor, II, R. M., Hudson, T. C., Seeger, A., Weber, H., Juliano, J., AND Helser, A. T. 2001. Vrpn: A deviceindependent, network-transparent vr peripheral system. In Proceedings of the ACM Symposium on Virtual Reality Software and Technology, ACM, New York, NY, USA, VRST '01, 55-61.

Tomioka, M., IKedA, S., ANd SATo, K. 2013. Approximated user-perspective rendering in tablet-based augmented reality. In Mixed and Augmented Reality (ISMAR), 2013 IEEE International Symposium on, 21-28.

Wagner, D., Pintaric, T., Ledermann, F., And SchmalSTIEG, D. 2005. Towards massively multi-user augmented reality on handheld devices. Springer.

Yoshida, T., Kuroki, S., NiI, H., KaWAKami, N., AND TACHI, S. 2008. Arscope. In ACM SIGGRAPH 2008 New Tech Demos, ACM, New York, NY, USA, SIGGRAPH '08, 4:1-4:1. 